\title{
RECURSIVE REAL NUMBERS
}

H. G. RICE ${ }^{1}$

A basic step in applying a new concept such as recursive functions to analysis should be an investigation of its application to the number system. We present here some of the elementary properties of recursive real numbers, as defined below.

A recursive real number may be described intuitively as one for which we can effectively generate as long a decimal expansion as we wish [5], or equivalently, to which we can effectively find as close a rational approximation as we wish. Accepting the thesis of Church [1, pp. 317-323], we interpret "effectiveness" (or the lack of it) as meaning the existence (or nonexistence) of certain recursive functions.

1. Definitions. The positive, zero, and negative rational numbers can be recursively enumerated by any constructive procedure for proving them denumerable. ${ }^{2} \mathrm{~A}$ rational number is then considered "given" if a corresponding integer is given. So to every sequence of non-negative integers corresponds a sequence of rational numbers, and conversely. We say that an infinite sequence of rational numbers is recursively enumerable (r.e.) if the corresponding sequence of integers is the sequence of values $g(0), g(1), g(2), \cdots$ of a general recursive function $g(x)$.

Definition. A r.e. sequence of rational numbers $a_{0}, a_{1}, a_{2}, \cdots$ is recursively convergent (r.c.) when there exists a general recursive function $g(x)$ such that $\left|a_{n}-a_{m}\right|<1 / N$ for $g(N)<n, m .^{3} g(x)$ will be called a convergence function for the sequence.

Definition A. A real number $a$ is a recursive real number when it is the limit of a r.e., r.c. sequence of rational numbers.

We mention three other definitions.

Definition B. $a$ is a recursive real number when the sign and the integral part of $a$ are given explicitly, and the digits of a binary expansion of the fractional part of $a$ are the sequence $g(0), g(1)$, $g(2), \cdots$ of values of a general recursive function $g(x) . g(x)$ is then the characteristic function of a recursive set.

Presented to the Society, September 1, 1953; received by the editors June 20, 1953 .

${ }^{1}$ This research was supported in part by the United States Air Force under Contract No. AF 18(600)-480 monitored by the Office of Scientific Research.

${ }^{2}$ See, e.g., Birkhoff and MacLane, Survey of modern algebra, rev. ed., New York, 1953, p. 359, Theorem 4.

${ }^{3}$ This inequality, like others we shall write, involves fractions. However, it is readily converted into an equivalent statement involving only non-negative integers. 
Definition C. $a$ is a recursive real number when the Dedekind sets of rational numbers associated with $a$ are recursive sets (i.e., the corresponding sets of integers are recursive).

Definition D. $a$ is a recursive real number when $a$ is contained in each of a r.e. sequence of nested intervals with rational end points, where the length of the intervals approaches zero.

All four definitions are equivalent. They were first mentioned by Specker [4], with the restriction that all pertinent functions be primitive recursive. Under this restriction they are not equivalent. However, Robinson [2] stated and outlined a proof of their equivalence where general recursive functions are allowed. ${ }^{4}$ The proof, except for A and B, will not be included here; the reader will not find it difficult to complete with the help of Theorem 1.

\section{Order.}

Theorem 1. Let $a_{0}, a_{1}, a_{2}, \cdots$ and $b_{0}, b_{1}, b_{2}, \cdots$ be r.e., r.c. sequences of rational numbers with limits $a$ and $b$ respectively, $a \neq b$. There exists an effective general method for deciding whether $a<b$ or $b<a$.

Proof. Since $a \neq b$, there exists an integer $n_{1}$ such that $|a-b|$ $>4 / n_{1}$. Let $f(x)$ and $g(x)$ be recursive convergence functions for $a_{0}, a_{1}, a_{2}, \cdots$ and $b_{0}, b_{1}, b_{2}, \cdots$ respectively. Then $n_{1}$ has the property that $2 / n_{1}<\left|a_{f\left(n_{1}\right)}-b_{g\left(n_{1}\right)}\right|$. For

$$
4 / n_{1}<|a-b| \leqq\left|a-a_{f\left(n_{1}\right)}\right|+\left|a_{f\left(n_{1}\right)}-b_{g\left(n_{1}\right)}\right|+\left|b_{g\left(n_{1}\right)}-b\right|
$$

and $\left|a-a_{f\left(n_{1}\right)}\right|,\left|b_{g\left(n_{1}\right)}-b\right| \leqq 1 / n_{1}$, so

$$
4 / n_{1}<\left|a_{f\left(n_{1}\right)}-b_{g\left(n_{1}\right)}\right|+2 / n_{1} .
$$

Define $n_{2}=\mu y\left[\left|a_{f(y)}-b_{g(y)}\right|>2 / y\right] . n_{2}$ exists and $n_{2} \leqq n_{1}$. Now $a-b$ $=a-a_{f\left(n_{2}\right)}+a_{f\left(n_{2}\right)}-b_{g\left(n_{2}\right)}+b_{g\left(n_{2}\right)}-b$, and since

$$
\left|a-a_{f\left(n_{2}\right)}\right|+\left|b_{g\left(n_{2}\right)}-b\right| \leqq 2 / n_{2}<\left|a_{f\left(n_{2}\right)}-b_{g\left(n_{2}\right)}\right|,
$$

then $a<b$ if and only if $a_{f\left(n_{2}\right)}<b_{g\left(n_{2}\right)}$.

In the case where $a_{i}$ and $b_{i}$ (hence $a$ and $b$ ) are non-negative, suppose $a_{i}=p_{a}(i) / q_{a}(i)$ and $b_{i}=p_{b}(i) / q_{b}(i)$, with the $p$ 's and $q$ 's recursive functions of $i$. The function

$$
1-\left(p_{a}\left(f\left(n_{2}\right)\right) \cdot q_{b}\left(g\left(n_{2}\right)\right)-p_{b}\left(g\left(n_{2}\right)\right) \cdot q_{a}\left(f\left(n_{2}\right)\right)\right)
$$

equals 1 when $a<b$ and 0 when $b<a$. Similar functions can be constructed for the cases involving negative values.

Theorem 2. Definitions A and B are equivalent.

${ }^{4}$ See also Myhill, J. Symbolic Logic vol. 18 (1953) pp. 7-10. 
Proof. Given a recursive binary expansion $b_{0} \cdot b_{1} b_{2} \cdots\left(b_{0}\right.$ an integer, $\left.b_{i+1}=0,1\right)$ of $a$, the sequence

$$
b_{0}, b_{0}+b_{1} / 2, b_{0}+b_{1} / 2+b_{2} / 4, \cdots
$$

is of the desired kind. Given the r.e., r.c. sequence of rational numbers $a_{0}, a_{1}, a_{2}, \cdots$ approaching $a$, the function

$$
\begin{gathered}
b(0)=\mu y[a<y]-1, \\
b(i+1)=1-\left(p_{b} \cdot q_{a}\left(f\left(n_{2}\right)\right)-q_{b} \cdot p_{a}\left(f\left(n_{2}\right)\right)\right),
\end{gathered}
$$

where $p_{b}=1+\sum_{y=0}^{i} 2^{i+1-y} \cdot b(y)$ and $q_{b}=2^{i+1}$, gives a binary expansion of $a$ if $a$ is irrational and non-negative. A similar function can be defined if $a$ is negative.

If $a=b$ the proof of Theorem 1 fails, since $n_{1}$ will not exist and the function giving $n_{2}$ will be undefined. This case is in fact undecidable.

THEOREM 3. There exists no effective general method for deciding whether or not the limit a of a r.e., r.c. sequence of rational numbers $a_{0}, a_{1}, a_{2}, \cdots$ is equal to zero.

Proof. If such a general method existed, it would enable us to solve the following problem. If $f(x)$ is a general recursive function whose range is known to be included in the set $\{0,1\}$, is $\{0\}$ the range of $f(x)$ ? For the sequence $f(0), f(0)+f(1) / 2, f(0)+f(1) / 2+f(2) / 4, \cdots$ is a r.e., r.c. sequence of rational numbers whose limit is 0 if and only if the range of $f(x)$ is $\{0\}$.

However, such a decision procedure for $f(x)$ cannot exist, as is well known. For let $h(x)$ be any general recursive function whose range is a r.e. set $\theta$. Then $1-|k-h(x)|$ is a general recursive function whose range is included in $\{0,1\}$, and whose range is $\{0\}$ if and only if $k \notin \theta$. So a decision procedure for our problem would give a decision procedure for every r.e. set.

COROLlary. There exists no effective general method for deciding, given two recursive real numbers $a$ and $b$ by r.e., r.c. sequences, whether or not $a=b$.

For otherwise we could solve the problem of the theorem by comparing the sequence $f(0), f(0)+f(1) / 2, f(0)+f(1) / 2+f(2) / 4, \cdots$ with the sequence $0,0,0, \cdots$. The corollary thus holds even if, say, $b$ is rational and given explicitly.

\section{The field of recursive real numbers.}

Lemma A. Every recursive real number a which is non-negative is the limit of a r.e., r.c. sequence of positive rational numbers, and every 
negative $a$ is the limit of such a sequence of negative rational numbers.

Proof. If $a=0$, then $1 / 2,1 / 3,1 / 4, \cdots$ is a sequence with the desired property.

If $0<a$, then the sequence obtained from any r.e., r.c. sequence approaching $a$ by dropping all nonpositive terms is a sequence with the desired property. Similarly for $a<0$.

We shall assume in this section that all sequences given are of the type of Lemma A.

Lemma B. Let $a_{0}, a_{1}, a_{2}, \cdots$ be a r.e., r.c. sequence of rational numbers. A rational number $b$ can be effectively found such that $\left|a_{i}\right|<b$ for all $i$. If the limit of $a_{0}, a_{1}, a_{2}, \cdots$ is not zero, a positive rational number $c$ can be effectively found such that $c<\left|a_{i}\right|$ for all $i$.

PROOF. If $f(x)$ is a convergence function for $a_{0}, a_{1}, a_{2}, \cdots$, set

$$
u(x, y)=\left\{\begin{array}{lll}
0 & \text { if } & x-\left|a_{f(y)}\right| \leqq 1 / y, \\
1 & \text { if } & x-\left|a_{f(y)}\right|>1 / y .
\end{array}\right.
$$

$u(x, y)$ can be defined recursively in two cases $\left(0<a_{i}\right.$ and $\left.a_{i}<0\right)$. Now

$$
b=\max \left[\left|a_{0}\right|,\left|a_{1}\right|, \cdots,\left|a_{f\left(y_{1}\right)}\right|, x_{1}\right],
$$

where $x_{1}=K(\mu z[u(K(z), L(z))=1]), y_{1}=L(\mu z[u(K(z), L(z))=1])$ and $K(z), L(z)$ are the Cantor pairing functions:

$\begin{array}{lllllllll}z & 0 & 1 & 2 & 3 & 4 & 5 & 6 & 7 \ldots \\ K(z) & 0 & 0 & 1 & 0 & 1 & 2 & 0 & 1 \ldots \\ L(z) & 0 & 1 & 0 & 2 & 1 & 0 & 3 & 2 \ldots .\end{array}$

The existence of $x_{1}$ and $y_{1}$ is guaranteed by the boundedness of any Cauchy sequence.

Suppose the limit of $a_{0}, a_{1}, a_{2}, \cdots$ is not zero. Set

$$
v(x, y)=\left\{\begin{array}{lll}
0 & \text { if } & \left|a_{f(y)}\right|-1 / x \leqq 1 / y, \\
1 & \text { if } & \left|a_{f(y)}\right|-1 / x>1 / y .
\end{array}\right.
$$

Then $c=\min \left[\left|a_{0}\right|,\left|a_{1}\right|, \cdots,\left|a_{f\left(y_{2}\right)}\right|, 1 / x_{2}\right]$, where

$$
x_{2}=K(\mu z[v(K(z), L(z))=1]) \text { and } y_{2}=L(\mu z[v(K(z), L(z))=1]) \text {. }
$$

THEOREM 4. The recursive real numbers form a field.

Proof. Suppose recursive real numbers $a, b, c$, with $c \neq 0$, given by r.e., r.c. sequences $a_{0}, a_{1}, a_{2}, \cdots, b_{0}, b_{1}, b_{2}, \cdots, c_{0}, c_{1}, c_{2}, \cdots$ respectively. Then evidently the r.e. sequences 


$$
\begin{aligned}
& a_{0}+b_{0}, a_{1}+b_{1}, a_{2}+b_{2}, \cdots, \\
& a_{0} \cdot b_{0}, a_{1} \cdot b_{1}, a_{2} \cdot b_{2}, \cdots, \\
& -a_{0},-a_{1},-a_{2}, \cdots, \\
& 1 / c_{0}, 1 / c_{1}, 1 / c_{2}, \cdots
\end{aligned}
$$

have the limits $a+b, a \cdot b,-a$, and $1 / c$ respectively. The only problem we need to consider is the recursive convergence. But with the bounds supplied by Lemma $B$, the standard proofs ${ }^{5}$ for the convergence of these sequences require no essential alteration for our purposes, and new convergence functions are readily defined recursively in terms of the bounds and the old convergence functions.

We shall designate the field of recursive real numbers by $\mathcal{E}$.

\section{Sequences.}

Definition. A sequence of recursive real numbers $a_{0}, a_{1}, a_{2}, \ldots$ is a r.e. sequence of recursive real numbers when there exist general recursive functions $f(x, y)$ and $g(x, y)$ such that $f(i, y)$ enumerates a r.c. sequence of rational numbers $a_{i, 0}, a_{i, 1}, a_{i, 2}, \cdots$ with $a_{i}$ as limit and $g(i, y)$ as convergence function.

The concept of recursive convergence can be extended without change to r.e. sequences of numbers of $\mathcal{E}$.

Theorem 5. Every r.e., r.c. sequence of numbers of $\mathcal{E}$ has a limit in $\mathcal{E}$.

Proof. To the notation of the above definition add $h(x)$ as a recursive convergence function for the sequence $a_{0}, a_{1}, a_{2}, \cdots$, and $a$ as its limit. Consider the sequence $a_{0,0}, a_{1, g(1,1)}, a_{2, g(2,2)}, \cdots$. We have $\left|a_{i, g(i, i)}-a_{j, g(j, j)}\right| \leqq\left|a_{i, g(i, i)}-a_{i}\right|+\left|a_{i}-a_{j}\right|+\left|a_{j}-a_{j, g(j, j)}\right|$. If $i, j \geqq \max [h(3 N), 3 N]$, then $\left|a_{i}-a_{j}\right|<1 / 3 N$ and

$$
\left|a_{k, o(k, k)}-a_{k}\right| \leqq 1 / k \leqq 1 / 3 N
$$

for $k=i, j$. So $\max [h(3 N), 3 N]$ is a recursive convergence function for the sequence, and similar consideration of $\left|a_{k, g(k, k)}-a\right|$ will show that $a$ is its limit. Hence $a \in \mathcal{E}$.

\section{Algebraic closure.}

Theorem 6. $\mathcal{E}(i)$ is algebraically closed.

Proof. We do not give a complete formal proof of this theorem. It would be a lengthy adaptation of the proof of Rosenbloom [3] of the fundamental theorem of algebra, with Rosenbloom's constructive

\footnotetext{
5 See, e.g., MacDuffee, Introduction to abstract algebra, New York, 1940, Chap. VI.
} 
procedures formalized as recursive functions. We content ourselves with indicating what adjustments must be made where other than rational operations are to be performed.

We suppose a polynomial $P(z)$ given by its degree and by r.e., r.c. sequences of rational numbers converging to the real and imaginary parts of its coefficients. For a value of $z$ similarly given, Theorem 4 asserts that we can obtain r.e., r.c. sequences approaching the real and imaginary parts of $P(z)$.

The first point in Rosenbloom's proof which needs discussion is in the definition preceding Lemma 1 . We must find the largest of a finite set of non-negative recursive real numbers. Let $\left\{a_{0}, a_{1}, \cdots, a_{N}\right\}$ be the set, with each $a_{j}$ given by a r.e. sequence $a_{j, 0}, a_{j, 1}, a_{j, 2}, \cdots$ of rational numbers and a recursive convergence function $f_{j}(x)$. Then the r.e. sequence $b_{k}=\max \left[a_{0, k}, a_{1, k}, \cdots, a_{N, k}\right]$ converges to $\max \left[a_{0}, a_{1}, \cdots, a_{N}\right]$ with convergence function $f(x)=\max \left[f_{0}(x)\right.$, $\left.f_{1}(x), \cdots, f_{N}(x)\right]$. For if $r$ is given, let $M=\max \left[f_{0}(r), f_{1}(r), \cdots\right.$, $\left.f_{N}(r)\right]$. Then if $M \leqq k, m$ we must show that $\left|b_{k}-b_{m}\right|<1 / r$. Let $b_{k}=a_{j_{k}, k}$ and $b_{m}=a_{j_{m}, m}$. If $b_{m} \leqq b_{k}$, we have

$$
\begin{array}{rlrl}
\left|b_{k}-b_{m}\right| & =b_{k}-b_{m}=a_{j_{k}, k}-a_{j_{k}, m}+a_{j_{k}, m}-a_{j_{m}, m} \\
& \leqq a_{j_{k}, k}-a_{j_{k}, m} & \quad\left(\text { since } a_{j_{k}, m}-a_{j_{m}, m} \leqq 0\right) \\
& <1 / r & \left(\text { since } f_{j_{k}}(r) \leqq M \leqq k, m\right),
\end{array}
$$

and similarly if $b_{k}<b_{m}$.

An analogous construction gives a sequence $c_{k}$ with the same convergence function and $\min \left[a_{0}, a_{1}, \cdots, a_{N}\right]$ as limit. This we can use after Corollary 1, where we must find, out of a finite set of nonnegative recursive real numbers, one which satisfies an inequality. Because of the possibility that $|P((k+i 1) a / n)|=\epsilon$ (we may take $\epsilon$ rational) for some members of the set, we cannot follow Rosenbloom's suggestion and simply check the numbers in order. If, however, we select the smallest of the set, clearly it will serve as $\boldsymbol{z}_{\epsilon}$.

Next in Lemma 3 we must define recursively functions $f\left(N, a_{0}, a_{1}, \cdots, a_{N}, \epsilon, x\right)$ which, considered as functions of $x$, have as range the numbers $z_{1}, z_{2}, \cdots, z_{N}$ of the lemma. Since the $a_{i}$ 's (the coefficients of $P(z)$ ) need not be rational, we must extend the concept of recursiveness to functions of recursive complex numbers. (The extension to functions of rational numbers has already been tacitly made.) We can say that $f(a)$ is recursive if, when $a_{0}, a_{1}, a_{2}, \cdots$ is a r.e., r.c. sequence of rational numbers converging to $a$, the sequence $f\left(a_{0}\right), f\left(a_{1}\right), f\left(a_{2}\right), \cdots$ is a r.e., r.c. sequence of numbers of $\boldsymbol{\varepsilon}$ converging to $f(a)$. For functions of complex numbers, we apply this to the 
real and imaginary parts. Now the argument of the preceding paragraph was to the effect that

$$
g\left(N, a_{0}, a_{1}, \cdots, a_{N}, \epsilon\right)=z_{\epsilon}
$$

is recursive. Set

$$
\begin{aligned}
f\left(1, a_{0}, a_{1}, \epsilon, x\right) & =-a_{0} / a_{1}, \\
f\left(N+1, a_{0}, a_{1}, \cdots, a_{N+1}, \epsilon, 0\right) & =g\left(N+1, a_{0}, a_{1}, \cdots, a_{N+1}, \epsilon\right), \\
f\left(N+1, a_{0}, a_{1}, \cdots, a_{N+1}, \epsilon, x+1\right) & =f\left(N, b_{0}, b_{1}, \cdots, b_{N}, \epsilon / C, x\right),
\end{aligned}
$$

where $b_{m}$ and $C$ are defined by Rosenbloom.

The final remark concerns the choice of sequence elements $z_{n}$ in Theorem 3. The situation is similar to that of Corollary 1, and a similar method handles it. This gives us the recursive enumerability of the sequence; Rosenbloom provides its recursive convergence. Hence the limit of the sequence (a root of the polynomial) has recursive real and imaginary parts, by our Theorem 5 .

6. Remarks. Theorem 6 is an illustration of the application of Theorem 5 in adapting the more constructive classical proofs to our purposes. It seems probable that another such adaptation ${ }^{6}$ would give $a^{b}$ in $\mathcal{E}(a, b$ in $\mathcal{E}, 0<a)$. If this is the case, it is unlikely that $\mathcal{E}$ will be criticized for the lack of any particular useful number. In fact, to "find" the value of a number (e.g., $\pi$, or Euler's constant) appears to be equivalent to giving a constructive proof that it is in $\mathcal{E}$, by Definition B.

On the other hand, from the point of view of theoretical analysis, $\mathcal{E}$ is not very satisfactory. Aside from its denumerability, which may or may not be a liability, we find, for example, that the following form of the Bolzano-Weierstrass theorem:

Every bounded, r.e. set of numbers of $\mathcal{E}$ has a limit point in $\mathcal{E}$. does not hold. For consider a r.e. but not recursive set $\theta$. Its characteristic function will correspond to a nonrecursive real number $b$. If $f(x)$ is a univalent general recursive function with $\theta$ as range, the sequence

$$
\frac{1}{2^{f(0)}}, \frac{1}{2^{f(0)}}+\frac{1}{2^{f(1)}}, \frac{1}{2^{f(0)}}+\frac{1}{2^{f(1)}}+\frac{1}{2^{f(2)}}, \cdots
$$

is a bounded, r.e. set of rational numbers with $b$ as its only limit point. ${ }^{7}$ The sequence is of course not recursively convergent.

${ }^{6}$ I have not carried out any formal work on this.

7 For a related result (differently interpreted) see [4]. 


\section{BIBLIOGRAPHY}

1. S. C. Kleene, Introduction to metamathematics, New York, Amsterdam, and Groningen, 1952.

2. R. M. Robinson, Review, J. Symbolic Logic vol. 16 (1951) p. 282.

3. P. C. Rosenbloom, An elementary constructive proof of the fundamental theorem of algebra, Amer. Math. Monthly vol. 52 (1945) pp. 562-570.

4. E. Specker, Nicht Konstructiv beweisbare Sätze der Analysis, J. Symbolic Logic vol. 14 (1949) pp. 145-158.

5. A. M. Turing, On computable numbers, with an application to the Entscheidungsproblem, Proc. London Math. Soc. (2) vol. 42 (1936-37) pp. 230-265.

UNIVERSITY OF NEW HAMPSHIRE

\section{A THEOREM ON HYPERSIMPLE SETS}

\section{J. C. E. DEKKER}

Introduction. E. L. Post proved in his paper Recursively enumerable sets of positive integers and their decision problems ${ }^{1}$ that a creative set cannot be reducible to a hypersimple set by truth tables (pp. 308310 ). The present paper is concerned with the question whether a creative set can be Turing reducible to a hypersimple set. It will be shown that the answer to this question is affirmative. In fact, we shall establish the following more general statement: for every recursively enumerable, but not recursive, set $\alpha$, a hypersimple set $\beta$ can be effectively found such that $\alpha$ and $\beta$ are Turing reducible to each other.

Preliminaries. A non-negative integer is called a number, a collection of numbers is called a set and a collection of sets a class. A set is immune, if it is infinite, but has no infinite recursively enumerable subset. For the definitions of a discrete array and a hypersimple set we refer to our paper Two notes on recursively enumerable sets ${ }^{2}$ (p. 497). We shall use the following notations: " $\rho(f)$ " for the range of the function $f(n)$, " $E$ " for the class of all recursive sets, " $F$ " for the class of all recursively enumerable (r.e.) sets, " $\alpha t$-red $\beta$ " for $\alpha$ is reducible to $\beta$ by truth tables, and " $\alpha$ red $\beta$ " for $\alpha$ is Turing reducible to $\beta$. If $a\left(t_{1}\right)$ and $a\left(t_{2}\right)$ are elements of the sequence $\{a(n)\}$, we say that $a\left(t_{2}\right)$ succeeds $a\left(t_{1}\right)$ in $\{a(n)\}$ if $t_{2}>t_{1}$.

Definition. Let $a(n)$ be a 1-1 function. The element $a(t)$ of the

\footnotetext{
1953.

Presented to the Society, November 27, 1953; received by the editors November 5 ,

1 Bull. Amer. Math. Soc. vol. 50 (1944) pp. 284-316.

2 Proc. Amer. Math. Soc. vol. 4 (1953) pp. 495-501.
} 